\title{
Apixaban for the Treatment of Japanese Subjects With Acute Venous Thromboembolism (AMPLIFY-J Study)
}

\author{
Mashio Nakamura, MD, PhD; Masakatsu Nishikawa, MD, PhD; Issei Komuro, $\mathrm{MD}, \mathrm{PhD}$; \\ Isao Kitajima, MD, PhD; Yoshio Uetsuka, MD, PhD; Takuji Yamagami, MD, PhD; \\ Hiroki Minamiguchi, MD, PhD; Rika Yoshimatsu, MD, PhD; Kosuke Tanabe; \\ Nobushige Matsuoka, PhD; Kazuhiro Kanmuri, PhD; Hisao Ogawa, MD, PhD
}

\begin{abstract}
Background: Anticoagulation is recommended as standard of care for venous thromboembolism (VTE) (pulmonary embolism [PE]/deep vein thrombosis [DVT]), for which unfractionated heparin (UFH) and warfarin are used in Japan. In the multi-regional AMPLIFY study, a fixed-dose regimen of apixaban alone was non-inferior to conventional therapy for treatment of PE/DVT and was associated with significantly fewer bleeding events.
\end{abstract}

Methods and Results: Japan phase 3 study (AMPLIFY-J), randomized, active-controlled, open-label study in Japanese subjects with acute PE/DVT, was designed based on AMPLIFY. Key objectives were to investigate safety and efficacy of apixaban in symptomatic PE/DVT subjects during 24-week treatment. UFH/warfarin was used as control treatment. Apixaban was initiated at $10 \mathrm{mg}$ twice daily for 7 days, followed by $5 \mathrm{mg}$ twice daily for 23 weeks. All endpoints and imaging for thrombotic burden were assessed by an event adjudication committee. Eighty subjects were randomized, 33 subjects $(41.3 \%)$ were aged $<65$ years. Proportion of major/clinically relevant non-major bleeding was lower in apixaban (7.5\%) compared with well-controlled UFH/warfarin (28.2\%; median TTR, 70.1\%). Recurrent VTE occurred in no subjects in apixaban and in 1 subject in UFH/warfarin. Thrombotic burden results were similar in both groups. Proportions of subjects with adverse events was generally similar in both groups.

Conclusions: Apixaban was well-tolerated and had a favorable safety profile. No clinically important efficacy difference compared with UFH/warfarin was observed. (Circ J 2015; 79: 1230-1236)

Key Words: Apixaban; Deep vein thrombosis; Japanese; Oral anticoagulant; Pulmonary embolism

$\mathbf{V}$ enous thromboembolism (VTE) is broadly divided into pulmonary embolism (PE) and deep vein thrombosis (DVT). PE and DVT have a common pathophysiology: PE is present in approximately $70 \%$ of DVT cases, and DVT is present in $>30-70 \%$ of PE cases when assessed on computed tomography (CT) or perfusion. ${ }^{1}$ The increasing westernization of the Japanese lifestyle, the aging population, and a greater rate of diagnosis associated with awareness of the disease have resulted in a greater number of deaths attributed to PE in recent years in Japan. ${ }^{2}$

Whereas the early diagnosis and treatment of VTE can result in relatively good outcome, $\mathrm{PE}$ is a fatal disease characterized by a high rate of mortality; indeed, sudden death may be the presenting symptom of VTE. ${ }^{3}$ Early diagnosis and early treatment, including inhibition of DVT progression and prevention of PE recurrence are therefore very important in the treatment

Received February 18, 2015; revised manuscript received April 3, 2015; accepted April 5, 2015; released online April 24, 2015 Time for primary review: 19 days

Department of Cardiology and Nephrology, Mie University Graduate School of Medicine, Tsu (M. Nakamura); Clinical Research Support Center, Mie University Hospital, Tsu (M. Nishikawa); Department of Cardiovascular Medicine, University of Tokyo Graduate School of Medicine, Tokyo (I. Komuro); Department of Clinical Laboratory and Molecular Pathology, Graduate School of Medical and Pharmaceutical Science, University of Toyama, Toyama (I. Kitajima); Department of Health Service and Hospital Administration, Tokyo Women's Medical University, Tokyo (Y.U.); Department of Radiology, Kochi Medical School, Kochi University, Nankoku (T.Y., R.Y.); Department of Radiology, Wakayama Medical University, Wakayama (H.M.); Clinical Research (K.T., K.K.), Clinical Statistics (N.M.), Development Japan, Pfizer Japan, Tokyo; Department of Cardiovascular Medicine, Graduate School of Medical Sciences, Kumamoto University, Kumamoto (H.O.); and National Cerebral and Cardiovascular Center, Suita (H.O.), Japan

This paper was presented at the $79^{\text {th }}$ Annual Scientific Meeting of the Japanese Circulation Society, Late Breaking Clinical Trials 1-5 (April 24, 2015, Osaka, Japan).

Mailing address: Mashio Nakamura, MD, PhD, Department of Cardiology and Nephrology, Mie University Graduate School of Medicine, 2-174 Edobashi, Tsu 514-8507, Japan. E-mail: mashio@clin.medic.mie-u.ac.jp

ISSN-1346-9843 doi:10.1253/circj.CJ-15-0195

All rights are reserved to the Japanese Circulation Society. For permissions, please e-mail: cj@j-circ.or.jp 
of VTE. ${ }^{2,4}$

Anticoagulant therapy is currently recommended by the Japanese Circulation Society as the standard of care for PE and DVT, for which unfractionated heparin (UFH) or fondaparinux, and warfarin are used in Japan. ${ }^{2}$ Dose adjustment, however, is a complicated matter when 2 drugs are used (warfarin with UFH or fondaparinux). The non-vitamin $\mathrm{K}$ antagonist oral anticoagulants (NOAC) have the potential to allow patients to be treated by monotherapy from acute to chronic phase, which is a long-awaited treatment option; and to replace conventional therapy for VTE. ${ }^{5}$

Apixaban is an orally administered reversible inhibitor that binds to blood coagulation activated factor X (FXa). ${ }^{5}$ AMPLIFY, a multi-regional, randomized, double-blind, active-controlled study in which Japanese subjects were not included, showed that a fixed-dose regimen of oral apixaban alone was as effective as conventional treatment consisting of enoxaparin followed by warfarin, and was associated with a clinically relevant reduction of $69 \%$ in major bleeding, indicating that apixaban can provide a simple, effective, and safe regimen for the initial and long-term treatment of VTE. ${ }^{6}$ Moreover, it would be of great interest to determine the health economics of NOAC for VTE, and several reports including from The National Institute for Health and Care Excellence indicated that apixaban could be considered a clinically and cost-effective option for VTE. ${ }^{7,8}$

In the AMPLIFY-J study, we investigated the safety and efficacy of apixaban in Japanese acute PE/DVT subjects with the primary composite endpoint of the adjudicated International Society on Thrombosis and Haemostasis (ISTH) major ${ }^{9}$ and clinically relevant non-major (CRNM) bleeding event. ${ }^{10}$

\section{Methods}

\section{Patients}

All subjects gave written informed consent prior to participation. Japanese patients, $\geq 20$ years of age and who had objectively confirmed, symptomatic proximal DVT or PE (with or without DVT) were enrolled in the study. Proximal DVT was defined as thrombosis involving at least the popliteal vein or a more proximal vein. In this study, safety evaluation was the primary objective, so that treatment with an inferior vena cava filter frequently performed in VTE treatment in Japan was permitted and added as a stratification factor for randomization.

Patients were excluded if they had thrombectomy or used fibrinolytic agent, had active bleeding, a high risk of bleeding, or other contraindications to treatment with UFH and warfarin; if they had another indication for long-term anticoagulation therapy, dual antiplatelet therapy, or treatment with aspirin $>165 \mathrm{mg}$ daily. Other key exclusion criteria were $>2$ doses of fondaparinux, or continuous infusion of UFH $>36 \mathrm{~h}$, and $>2$ doses of oral vitamin $\mathrm{K}$ antagonist before first administration of the study drug. Additional exclusion criteria were hemoglobin $<9 \mathrm{~g} / \mathrm{dl}$, platelet count $<100,000 / \mathrm{mm}^{3}$, and creatinine clearance $<25 \mathrm{ml} / \mathrm{min}$.

\section{Study Design}

AMPLIFY-J, a randomized, active-controlled, open-label study in 80 Japanese subjects with acute PE/DVT, was based on the design for the AMPLIFY study. The key objectives were to investigate the safety and efficacy of apixaban in Japanese symptomatic proximal DVT and PE subjects for the 24-week treatment period. Given that enoxaparin (low-molecularweight heparin) does not have an indication for VTE treatment in Japan, the conventional therapy of UFH/warfarin for the control group was used. Using an interactive voice response system, subjects were randomized to either UFH/warfarin or apixaban at a 1:1 ratio based on the type of index event (symptomatic proximal DVT or symptomatic PE) and the use of a caval filter.

Patients assigned to UFH/warfarin were given a continuous i.v. infusion of UFH so as to maintain the activated partial thromboplastin time in the range 1.5-2.5-fold the control value. Warfarin was also concomitantly administered. UFH was continuously given until the effect of warfarin was stabilized; after which, subjects were given warfarin alone. UFH was given for at least 5 days consecutively and was discontinued at once if the prothrombin time-international normalized ratio (PT-INR) was $\geq 1.5$. If PT-INR exceeded 2.0 within the initial 5 days of administration, UFH could be discontinued based on the investigator's judgment. The warfarin dose was adjusted to maintain INR between 1.5 and 2.5 in accordance with Japan PE/DVT treatment guidelines. ${ }^{2}$ With regard to the apixaban group, subjects received $10 \mathrm{mg}$ b.i.d. apixaban for 7 days as an initial therapy, followed by $5 \mathrm{mg}$ b.i.d. apixaban for 23 weeks as long-term therapy; the same treatment regimen within the AMPLIFY study ${ }^{6}$ was supported by the dose-finding study in DVT subjects (BOTTICELLI ${ }^{11}$ ) and dose-response modeling.

The study was conducted according to the Declaration of Helsinki and in compliance with the International Conference on Harmonization and Good Clinical Practice guidelines. All local regulatory requirements and sponsoring company policies were followed. A data safety monitoring committee advised the study steering committee and study sponsor.

\section{Study Endpoints}

The primary endpoint of this study was the incidence of the adjudicated composite of ISTH-defined major bleeding ${ }^{9}$ and CRNM bleeding during the treatment period. Secondary safety endpoints were the incidence of the adjudicated ISTH major bleeding events and all bleeding events (ISTH major, CRNM, and minor) during the treatment period. With regard to efficacy endpoints, incidence of the following endpoints was investigated: composite endpoint of adjudicated recurrent symptomatic VTE (non-fatal DVT or non-fatal PE) or VTE-related death during 24 weeks; and thrombotic burden deterioration at 2, 12 and 24 weeks throughout the 24 -week treatment period. CT venography (CTV) and compression ultrasound (CUS) were used for assessment of DVT subjects, and CT pulmonary angiogram (CTPA) was used for PE subjects for thrombotic burden assessment. Imaging obtained at weeks 2, 12 and 24 (week 12 only in CTV for DVT subjects) were compared with imaging at week $0 /$ day 1 . The results were classified as improved, worsened, or no change.

All clinical endpoints (bleeding-related events, recurrent PE/DVT) and imaging data for thrombotic burden assessments were adjudicated by an event adjudication committee who were blinded to study drug assignment. Bleeding events were defined using ISTH criteria. The definition of CRNM bleeding was acute clinically overt bleeding, meeting one of the criteria described in Table S1. Minor bleeding was defined as acute clinically overt events not meeting either major or CRNM bleeding criteria. Definitions of recurrent symptomatic VTE/VTErelated death are given in Table S1, and detailed definitions of thrombotic burden assessment are described in the event adjudication committee charter.

\section{Statistical Analysis}

Treatment period was defined as the period from first dose through 2 days after discontinuation of the study drug (30 days after discontinuation of the study drug for serious adverse 


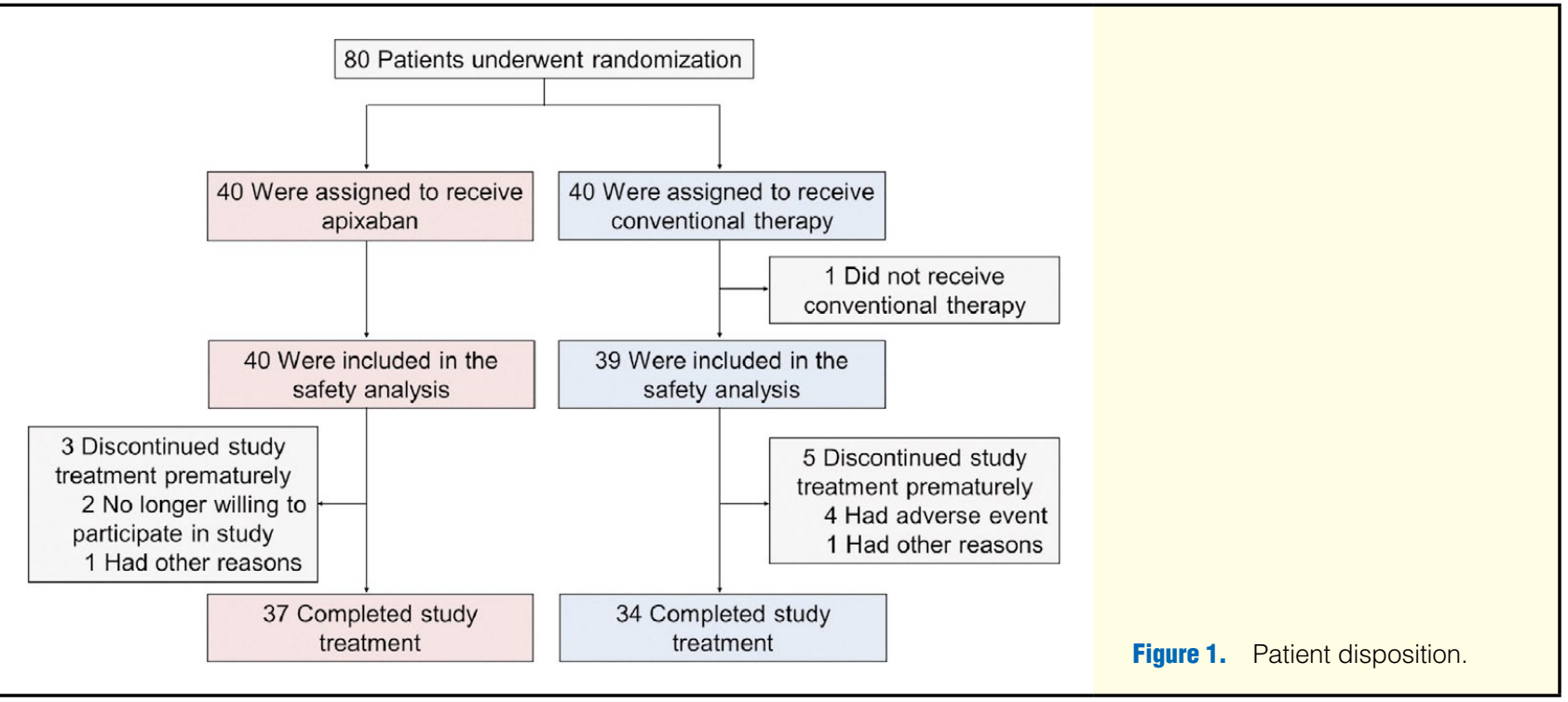

events [SAE]). Intended treatment period was defined as day of randomization until day 168 or 2 days after the last dose of the study drug, whichever was later, and up to day 168 for the subject who did not take the study drug. Follow-up period was defined as starting after the end of the treatment period and extending through 30 days after the last dose of the study drug.

The full analysis set (FAS) consisted of all of the subjects who were randomized (randomized subjects). The efficacy analysis was performed for subjects in FAS for whom outcome status during 24 weeks was documented. The comprehensive endpoint based on recurrence of symptomatic VTE and VTErelated death was calculated for the number of subjects in whom the event occurred, as well as the incidence thereof and 95\% confidence interval (CI). The evolution of thrombus during the study was evaluated in accordance with the categories "improved", "no change", and "worsened". The number and proportion of subjects in each category at weeks 2,12 , and 24 were calculated for each treatment group.

The safety analysis set included all of the subjects who received study treatment (at least once after randomization: treated subjects). The primary and secondary endpoints concerning bleeding events were analyzed for the number of subjects, as well as the proportion thereof and its 95\% CI. For the primary endpoint, the relative risk, 95\% CI and P-value were calculated as post-hoc analysis. The proportion of time in the therapeutic range (TTR) for INR 1.5-2.5 was calculated using the Rosendaal linear interpolation method, excluding the initial UFH treatment period and the time period when study warfarin dosing was temporarily interrupted. ${ }^{12}$ AE type was encoded in accordance with the system organ class and preferred terms of the ICH Medical Dictionary for Regulatory Activities (MedDRA) version 17.0. Change in clinical laboratory parameters from baseline to each visit was analyzed for each treatment group. Change in vital signs from baseline to each visit was also analyzed for each treatment group.

\section{Results}

\section{Patient Disposition and Baseline Characteristics}

This study was conducted at 21 sites in Japan. As shown in Figure 1, a total of 80 subjects were randomized (apixaban, 40 subjects; UFH/warfarin, 40 subjects). Of these, 1 subject randomized to UFH/warfarin was withdrawn from the study prior to the initiation of study treatment. A total of 9 subjects $(11.3 \%)$ discontinued from the treatment period; $3(7.5 \%)$ were in the apixaban group, and $6(15.0 \%)$ were in the UFH/ warfarin group. The proportion of subjects who discontinued due to $\mathrm{AE}$ was lower in the apixaban group (0 subjects) compared with the UFH/warfarin group (4/40, 10.0\%).

Baseline characteristics are listed in Table 1. A total of 33 subjects $(41.3 \%)$ were aged $<65$ years. The proportion of male subjects was slightly higher in the apixaban group compared with the UFH/warfarin group; this was reflected in the mean body weight, which was higher in the apixaban group. Most of subjects had normal or mild renal impairment in both treatment groups. Number of subjects with a caval filter at baseline was 5 in the apixaban group and 6 in the UFH/warfarin group.

\section{Treatment Compliance and INR Control}

In the apixaban group, compliance to study treatment was $\geq 80 \%$ in all subjects. In the UFH/warfarin group, the median proportion of TTR for INR 1.5-2.5 was 70.1\%.

\section{Primary Bleeding Endpoint}

The results of adjudicated bleeding endpoints during the treatment period are given in Table 2 . The proportion of subjects with major or CRNM bleeding during the treatment period (primary endpoint) was lower in the apixaban group (7.5\%) compared with the UFH/warfarin group (28.2\%). With regard to 2 major bleeds in the UFH/warfarin group, 1 subject (female, 47 years old, $80 \mathrm{~kg}$ ) who had menorrhagia, also developed recurrent $\mathrm{PE}$, and another subject (female, 66 years old, $48.5 \mathrm{~kg}$ ) had a thalamus hemorrhage. Dosage and INR at onset of each event were not collected. The proportions of every other bleeding endpoint (major, CRNM, minor and all bleeding events) during the treatment period were also lower in the apixaban group compared with the UFH/warfarin group. Subgroup analysis results by index event strata (DVT or PE) were generally consistent with overall study results.

\section{Recurrent VTE/NTE-Related Death}

No subject had recurrent VTE/VTE-related death in the apixaban group. Only 1 subject had recurrent PE during the intended treatment period, and there was no VTE-related death in the 


\begin{tabular}{|c|c|c|c|}
\hline & $\begin{array}{c}\text { Apixaban } \\
(n=40)\end{array}$ & $\begin{array}{l}\text { UFH/Warfarin } \\
\quad(n=40)\end{array}$ & $\begin{array}{l}\text { Total } \\
(n=80)\end{array}$ \\
\hline \multicolumn{4}{|l|}{ Index event strata } \\
\hline DVT & $22(55.0)$ & $23(57.5)$ & $45(56.3)$ \\
\hline $\mathrm{PE}$ & $18(45.0)$ & $17(42.5)$ & $35(43.8)$ \\
\hline \multicolumn{4}{|l|}{ Age (years) } \\
\hline Mean \pm SD & $64.3 \pm 13.40$ & $66.1 \pm 17.72$ & $65.2 \pm 15.64$ \\
\hline$<65$ & $18(45.0)$ & $15(37.5)$ & 33 (41.3) \\
\hline$\geq 65$ to $<75$ & $14(35.0)$ & $10(25.0)$ & $24(30.0)$ \\
\hline$\geq 75$ & $8(20.0)$ & $15(37.5)$ & $23(28.8)$ \\
\hline \multicolumn{4}{|l|}{ Gender } \\
\hline Male & $22(55.0)$ & $17(42.5)$ & $39(48.8)$ \\
\hline Female & $18(45.0)$ & $23(57.5)$ & $41(51.3)$ \\
\hline \multicolumn{4}{|l|}{ Weight (kg) } \\
\hline Mean \pm SD & $64.64 \pm 12.855$ & $58.14 \pm 12.208$ & $61.39 \pm 12.879$ \\
\hline$\leq 60$ & $17(42.5)$ & $26(65.0)$ & $43(53.8)$ \\
\hline$>60$ & $23(57.5)$ & $14(35.0)$ & 37 (46.3) \\
\hline \multicolumn{4}{|l|}{ BMI $\left(\mathrm{kg} / \mathrm{m}^{2}\right)$} \\
\hline Mean \pm SD & $24.39 \pm 2.999$ & $22.94 \pm 3.909$ & $23.67 \pm 3.538$ \\
\hline$\leq 25$ & $25(62.5)$ & 27 (67.5) & $52(65.0)$ \\
\hline$>25$ to $\leq 30$ & $13(32.5)$ & $11(27.5)$ & $24(30.0)$ \\
\hline$>30$ to $\leq 35$ & $2(5.0)$ & $2(5.0)$ & $4(5.0)$ \\
\hline$>35$ & 0 & 0 & 0 \\
\hline \multicolumn{4}{|l|}{ Renal impairment } \\
\hline Normal $(\mathrm{CrCL}>80 \mathrm{ml} / \mathrm{min})$ & $20(50.0)$ & $14(35.0)$ & $34(42.5)$ \\
\hline Mild $(50<\mathrm{CrCL} \leq 80 \mathrm{ml} / \mathrm{min})$ & $18(45.0)$ & $17(42.5)$ & $35(43.8)$ \\
\hline Moderate $(30<\mathrm{CrCL} \leq 50 \mathrm{ml} / \mathrm{min})$ & $1(2.5)$ & $5(12.5)$ & $6(7.5)$ \\
\hline Severe $(\mathrm{CrCL} \leq 30 \mathrm{ml} / \mathrm{min})$ & $1(2.5)$ & $3(7.5)$ & $4(5.0)$ \\
\hline Not reported & 0 & $1(2.5)$ & $1(1.3)$ \\
\hline Previous DVT & $6(15.0)$ & $4(10.0)$ & $10(12.5)$ \\
\hline Diabetes mellitus & $4(10.0)$ & $5(12.5)$ & $9(11.3)$ \\
\hline Hypertension & $19(47.5)$ & $23(57.5)$ & $42(52.5)$ \\
\hline Prior anti-coagulant therapy & $32(80)$ & $40(100)$ & $72(90.0)$ \\
\hline
\end{tabular}

Data given as mean \pm SD or $n(\%)$. BMI, body mass index; $\mathrm{CrCL}$, creatinine clearance; DVT, deep vein thrombosis; $\mathrm{PE}$, pulmonary embolism; UFH, unfractionated heparin; VKA, vitamin $\mathrm{K}$ antagonist.

\begin{tabular}{|c|c|c|c|c|c|c|}
\hline \multicolumn{7}{|c|}{ Index event: DVT } \\
\hline & $\begin{array}{c}\text { Apixaban } \\
(n=40)\end{array}$ & $\begin{array}{c}\text { UFH/WF } \\
(n=39)\end{array}$ & $\begin{array}{c}\text { Apixaban } \\
(n=22)\end{array}$ & $\begin{array}{c}\text { UFH/WF } \\
(n=22)\end{array}$ & $\begin{array}{c}\text { Apixaban } \\
(n=18)\end{array}$ & $\begin{array}{c}\text { UFH/WF } \\
(n=17)\end{array}$ \\
\hline \multicolumn{7}{|c|}{ Major or CRNM bleed } \\
\hline $\mathrm{n} / \mathrm{N}(\%)$ & $3 / 40(7.50)$ & $11 / 39(28.21)$ & $0 / 22$ & $5 / 22(22.73)$ & $3 / 18(16.67)$ & $6 / 17(35.29)$ \\
\hline $95 \% \mathrm{Cl}$ & $1.88-20.57$ & $16.42-43.90$ & $0.00-17.55$ & $9.71-43.85$ & $5.01-40.05$ & $17.17-58.84$ \\
\hline \multicolumn{7}{|c|}{ Major bleed } \\
\hline $\mathrm{n} / \mathrm{N}(\%)$ & $0 / 40$ & 2/39 (5.13) & $0 / 22$ & $0 / 22$ & $0 / 18$ & $2 / 17(11.76)$ \\
\hline $95 \% \mathrm{Cl}$ & $0.00-10.44$ & $0.52-17.79$ & $0.00-17.55$ & $0.00-17.55$ & $0.00-20.67$ & $2.03-35.59$ \\
\hline \multicolumn{7}{|c|}{ CRNM bleed } \\
\hline $\mathrm{n} / \mathrm{N}(\%)$ & $3 / 40(7.50)$ & 9/39 (23.08) & $0 / 22$ & $5 / 22(22.73)$ & $3 / 18(16.67)$ & $4 / 17(23.53)$ \\
\hline $95 \% \mathrm{Cl}$ & $1.88-20.57$ & $12.44-38.54$ & $0.00-17.55$ & $9.71-43.85$ & $5.01-40.05$ & $9.05-47.77$ \\
\hline \multicolumn{7}{|c|}{ Minor bleed } \\
\hline $\mathrm{n} / \mathrm{N}(\%)$ & $6 / 40(15.00)$ & $10 / 39(25.64)$ & 2/22 (9.09) & $3 / 22(13.64)$ & 4/18 (22.22) & $7 / 17$ (41.18) \\
\hline $95 \% \mathrm{Cl}$ & $6.68-29.46$ & $14.41-41.24$ & $1.34-29.00$ & $3.90-34.18$ & $8.47-45.75$ & $21.56-64.05$ \\
\hline \multicolumn{7}{|l|}{ All bleeds } \\
\hline $\mathrm{n} / \mathrm{N}(\%)$ & $7 / 40(17.50)$ & $17 / 39$ (43.59) & 2/22 (9.09) & 7/22 (31.82) & $5 / 18(27.78)$ & 10/17 (58.82) \\
\hline $95 \% \mathrm{Cl}$ & $8.43-32.26$ & $29.29-59.03$ & $1.34-29.00$ & $16.19-52.85$ & $12.17-51.20$ & $35.95-78.44$ \\
\hline
\end{tabular}

A single patient may report more than 1 bleeding event. $95 \% \mathrm{Cl}$ of incidence was calculated using the Agresti-Coull method. Overall bleeding includes major bleeding, CRNM bleeding, and minor bleeding. $\mathrm{Cl}$, confidence interval; CRNM, clinically relevant non-major; WF, warfarin. Other abbreviations as in Table 1. 
A) Thrombotic burden assessments based on CUS in DVT subjects

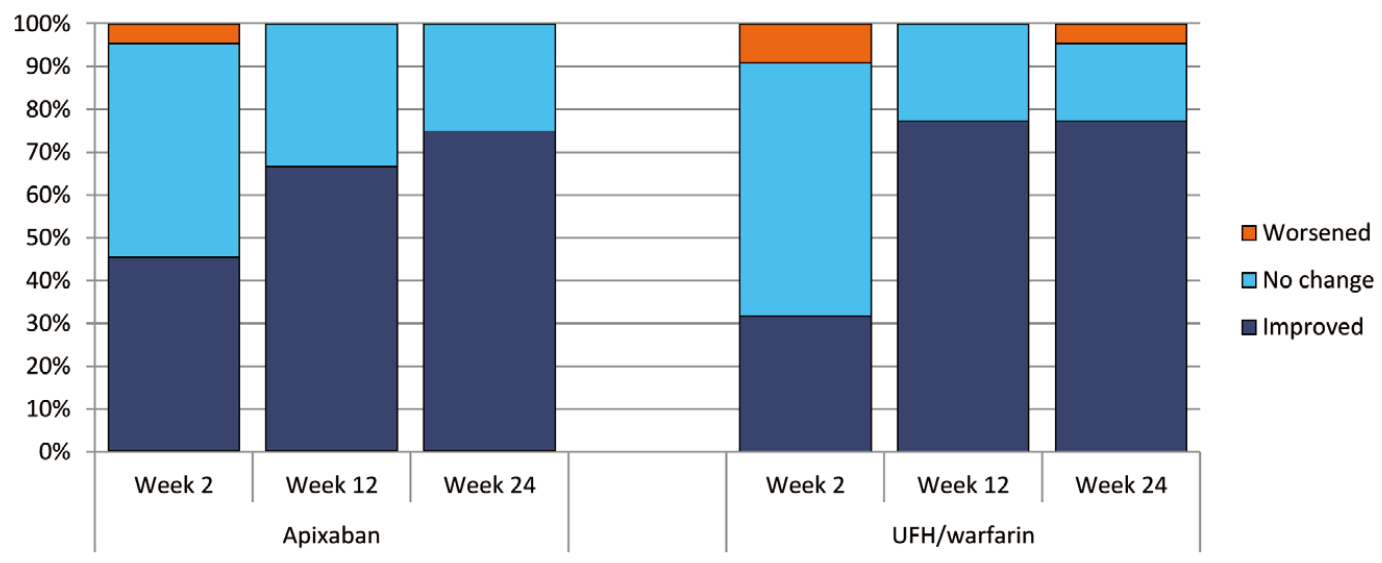

B) Thrombotic burden assessments based on CTPA in PE subjects
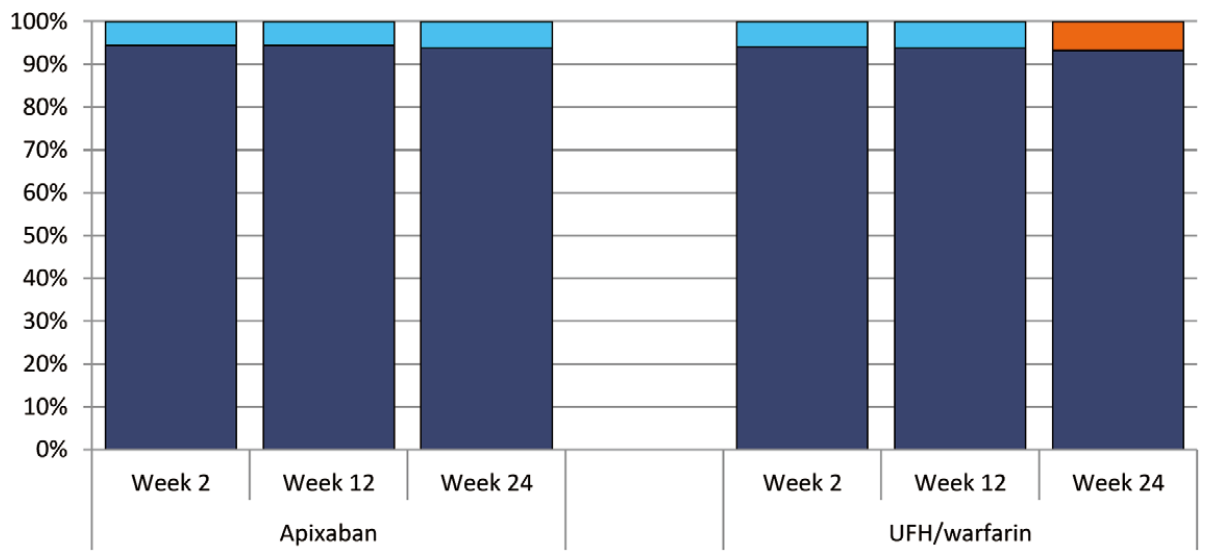

$\square$ Worsened

$\square$ No change

$\square$ Improved

Figure 2. Adjudicated thrombotic burden assessment: randomized subjects, based on (A) compression ultrasound (CUS) in deep vein thrombosis (DVT) subjects and (B) computed tomography pulmonary angiogram (CTPA) in pulmonary embolism (PE) subjects. Worsened, newly developed or clearly and significantly extended thrombi observed in lung or lower limb compare to baseline; improved, totally dissolved or clearly and significantly reduced thrombi observed in lung or lower limb compare to baseline; no change, neither worsened nor improved.

UFH/warfarin group. This subject with the index event of PE, temporarily stopped the study treatment (warfarin) on day 138 due to menorrhagia. Then, the patient developed worsening of PE on day 156. Study treatment was never restarted after day 138 and permanently discontinued due to the recurrent PE.

\section{Thrombotic Burden Assessment}

The number of subjects with DVT whose thrombus was determined as worsened on CUS at week 2 was $1(4.5 \%)$ in the apixaban group. The number of DVT subjects in the UFH/ warfarin group whose thrombus was determined to have worsened at weeks 2,12 , and 24 (or discontinuation) was $2(9.1 \%)$, 0 , and $1(4.5 \%)$, respectively, on CUS. The number of subjects with DVT whose thrombus was determined as improved on CUS at week 2 was $10(45.5 \%)$ in the apixaban group and 7 $(31.8 \%)$ in the UFH/warfarin group. The number of subjects who had improved on CUS assessment at week 12 was 14 $(66.7 \%)$ in the apixaban group and $17(77.3 \%)$ in the UFH/ warfarin group, and the CUS results at week 24 (or discontinu- ation) of treatment were similar to those at week 12 (Figure 2). According to week $12 \mathrm{CTV},>90 \%$ of subjects had improvement in both treatment groups.

In the apixaban group, no subjects with PE were assessed as worsened on CTPA, and there was 1 subject $(6.7 \%)$ in the UFH/warfarin group who was categorized as worsened at the end of treatment. All subjects had early improvement at week 2 in both treatment groups, except for 1 subject each. CTPA at weeks 12 and 24 (or discontinuation) of treatment was similar to that at week 2 (Figure 2). No clinically important difference between treatment groups in thrombotic burden assessment was observed.

\section{AE}

Adverse events during the treatment period are listed in Table 3. No death was reported in either of the present treatment groups. SAE were reported in 3 subjects $(7.5 \%)$ in the apixaban group and in 7 subjects $(17.9 \%)$ in the UFH/warfarin group. There were no AE resulting in discontinuation of study treatment in 
Table 3. AE During the Treatment Period: Treated Subjects

$\begin{array}{lcc} & \begin{array}{c}\text { Apixaban } \\ (\mathbf{n}=\mathbf{4 0})\end{array} & \begin{array}{c}\text { UFH/warfarin } \\ (\mathbf{n}=\mathbf{3 9})\end{array} \\ \text { All subjects } & & 39 \\ \text { Evaluable subjects } & 40 & 37(94.9) \\ \text { Subjects in whom AE occurred } & 34(85.0) & 7(17.9) \\ \text { Subjects in whom SAE occurred } & 3(7.5) & 1(2.6) \\ \text { Subjects in whom severe AE occurred } & 1(2.5) & 4(10.3) \\ \text { Subjects who discontinued treatment due to AE } & 0 & 4(10.3) \\ \text { Subjects who reduced dosage or discontinued treatment due to AE } & 4(10.0) & \end{array}$

Data given as $n(\%)$. AE, adverse event; SAE, serious adverse event. Other abbreviation as in Table 1.

the apixaban group; and 4 subjects (10.3\%) in the UFH/warfarin group reported a total of $5 \mathrm{AE}$ resulting in discontinuation of study treatment (unstable angina, gastric cancer, thalamus hemorrhage, PE, and DVT), in which thalamus hemorrhage was considered treatment related. The proportions of subjects with AE were generally similar in both groups (Table 3 ). In both treatment groups, $>80 \%$ of subjects had at least $1 \mathrm{AE}$ and most of the AE were mild or moderate in severity.

Treatment-related AE occurred in the treatment period in 13 subjects $(32.5 \%)$ in the apixaban group and in 9 subjects $(23.1 \%)$ in the UFH/warfarin group. The most frequently reported treatment-related AE were nasal bleeding (3 subjects; $7.5 \%$ ), abnormal liver function test, PE, and hematoma (2 subjects each; $5.0 \%)$ in the apixaban group, and subcutaneous hemorrhage ( 3 subjects; $7.7 \%$ ) in the UFH/warfarin group. All treatment-related AE were mild or moderate in severity, except 3 $\mathrm{AE}$ reported in 1 subject in the apixaban group (peripheral edema, subcutaneous hemorrhage, skin erosion).

\section{Discussion}

Anticoagulant therapy is currently recommended as the standard of care for VTE, for which UFH or fondaparinux, and warfarin are used in Japan. In the multi-regional AMPLIFY trial, apixaban was shown to be non-inferior for the treatment of recurrent VTE/VTE-related death and was statistically superior in the primary safety endpoint of major bleeding vs. standard of care. ${ }^{6}$ Whether apixaban can be an alternative to standard of care also in Japanese patients with VTE was unclear. To investigate the safety and efficacy of apixaban in Japanese symptomatic VTE (proximal DVT and PE) subjects during a 24-week treatment period, the AMPLIFY-J study was conducted.

Important findings of the study are that apixaban was welltolerated, had a favorable safety profile, and no clinically important difference in efficacy was observed, compared with wellcontrolled standard of care; UFH/warfarin had median TTR $70.1 \%$ for the recommended INR range in the Japan VTE treatment guideline. In addition, although the present sample size was not large, safety and efficacy of apixaban were consistent with overall results across predefined subgroups, including index event (PE and DVT), use of vena cava filter, age, weight, sex, renal function, and use of parenteral anticoagulant treatment before study drug administration. Furthermore, efficacy and safety of apixaban in Japanese VTE patients were consistent with the results of the multi-regional AMPLIFY study. In this study, target INR range (1.5-2.5) for warfarin treatment was lower than that in the AMPLIFY study (2.0-3.0), but still there were fewer bleeding events on apixaban than on conventional therapy.

This study was designed based on the AMPLIFY study, with the aim to determine whether we could extrapolate overseas data into a Japanese dataset based on the International Conference on Harmonization (ICH)-E5 guidelines. ${ }^{13}$ Characteristics of the study are as follows: first, the sequence of the clinical conditions and the recommended therapy were the same for both DVT and PE, and the AMPLIFY-J was designed to assess the efficacy and safety of apixaban in subjects with PE and DVT collectively. The incidence of major bleeding/CRNM, for each of the PE and DVT subgroups, was lower in the apixaban group than in the control group, and similar to those in the total subject group (Table 2). Also, symptomatic recurrent VTE/VTE-related death occurred only in 1 patient in the UFH/ warfarin group for the PE subgroup, and imaging assessment of thrombi showed similar results for the PE or DVT subgroup (Figure 2).

Second, the AMPLIFY-J study has shown that the higher dose of apixaban for acute treatment in Japanese VTE patients is clinically acceptable. The regimen for acute treatment is similar to that for fondaparinux, which is commercially available in Japan for both prevention of VTE and VTE treatment indications, and which has been approved at $2.5 \mathrm{mg}$ for prevention and $7.5 \mathrm{mg}$ for treatment. These dose regimens reflect the fact that risk of recurrence of VTE is the highest in the early stage of treatment and gradually decreases with time. Moreover, the safety profile of apixaban in a 24-week treatment period in Japanese acute VTE subjects was confirmed, and no clinically important difference was observed in efficacy profile of apixaban from that of UFH/warfarin therapy. Given that the current recommended duration of anticoagulation therapy for idiopathic VTE and congenital coagulation disorder in Japanese guidelines is "at least 3 months", AMPLIFY-J provides further clinical information. Results from the AMPLIFY study, clearly demonstrating the effectiveness and superior bleeding profile of apixaban $10 \mathrm{mg}$ b.i.d. for 7 days followed by $5 \mathrm{mg}$ b.i.d. compared with standard therapy, support the appropriateness of the apixaban treatment regimen for VTE.

Last, thrombotic burden assessment was conducted as a secondary efficacy endpoint in addition to recurrent VTE/VTErelated death, so as to fully evaluate the efficacy of apixaban compared with standard therapy. The timing of the assessment of thrombotic burden based on the Japanese guidelines ${ }^{2}$ and BOTTICELLI study; ${ }^{11}$ after completion of high-dose treatment (week 2), after completion of 3 months of treatment (week 12), and at study completion (week 24). There was no difference in thrombus evaluation between apixaban and UFH/warfarin (Figure 2). Moreover, inferior vena cava filter is considered an adjunct to anticoagulant therapy in the Japanese guidelines. ${ }^{2}$ Of 5 subjects with a caval filter in the apixaban group at baseline, no recurrence of VTE or deterioration in thrombotic burden assessment were observed in this study. 
There are limitations in this study. First, the number of subjects in this study was set at 80 based on feasibility, because of the relatively small number of DVT and PE subjects diagnosed in the clinical setting in Japan. Despite the small sample size, the proportion of major/CRNM bleeding was significantly lower in apixaban compared with UFH/warfarin (RR, 0.27; 95\% CI: $0.08-0.88 ; \mathrm{P}=0.0160$ ) on post-hoc analysis. With regard to efficacy, although the primary endpoint of VTE/VTE-related death was very low in this study, thrombotic burden assessment indicated that similar efficacy of apixaban to conventional therapy was expected (Figure 2). Second, this was an open label study, but the primary endpoint was predefined based on the well-recognized and validated ISTH definition, ${ }^{9}$ and all outcome events were adjudicated by an event adjudication committee in a blinded manner so as to maintain validity of assessment.

Overall, apixaban was well-tolerated, had a favorable safety profile, and no clinically important efficacy difference compared with UFH/warfarin was observed. Given that the present efficacy and safety of apixaban were consistent with those in the multi-regional AMPLIFY study, clinical data of apixaban obtained in overseas studies such as BOTTICELLI, AMPLIFY and AMPLIFY-EXT ${ }^{14}$ can be extrapolated to Japanese VTE patients.

\section{Disclosures}

M. Nakamura has received remuneration from Daiichi Sankyo, Bayer Yakuhin. M. Nishikawa has received remuneration and research funds from Daiichi Sankyo. I. Komuro has received remuneration from Daiichi Sankyo, Nippon Boehringer Ingelheim, and scholarship funds from Astellas Pharma, Daiichi Sankyo, Takeda Pharmaceutical, Nippon Boehringer Ingelheim, Bristol-Myers Squibb. I. Kitajima has received remuneration from Nippon Boehringer Ingelheim. H.O. has received remuneration from AstraZeneca, Bayer Yakuhin, Boehringer Ingelheim Japan, Bristol-Myers Squibb, Daiichi Sankyo, Mitsubishi Tanabe Pharma, MSD, Pfizer Japan, Sanofi, Takeda Pharmaceutical and Teijin Pharma, and has received research funds from Bayer Yakuhin, Daiichi Sankyo and Novartis Pharma, and has received scholarship funds from Astellas Pharma, AstraZeneca, Bristol-Myers Squibb, Chugai Pharmaceutical, Daiichi Sankyo, Dainippon Sumitomo Pharma, Kowa, MSD, Otsuka Pharmaceutical, Pfizer Japan, Sanofi, Shionogi and Takeda Pharmaceutical. Y.U., T.Y., H.M., R.Y. have no conflict of interest.

This study was funded by Pfizer Inc and Bristol-Myers Squibb.

\section{References}

1. Gómez-Outes A, Suárez-Gea ML, Lecumberri R, Terleira-Fernández AI, Vargas-Castrillón E. Direct oral anticoagulants in the treatment of venous thromboembolism, with a focus on patients with pulmonary embolism: An evidence-based review. Vasc Health Risk Manag 2014; 10: 627-639.

2. JCS Joint Working Group. Guidelines for the diagnosis, treatment and prevention of pulmonary thromboembolism and deep vein thrombosis (JCS 2009): Digest version. Circ J 2011; 75: 1258-1281.

3. Ota M, Nakamura M, Yamada N, Yazu T, Ishikura K, Hiraoka N, et al. Prognostic significance of early diagnosis in acute pulmonary thromboembolism with circulatory failure. Heart Vessels 2002; 17: 7-11.

4. Nakamura M, Miyata T, Ozeki Y, Takayama M, Komori K, Yamada $\mathrm{N}$, et al. Current venous thromboembolism management and outcomes in Japan. Circ J 2014; 78: 708-717.

5. Yeh CH, Gross PL, Weitz JI. Evolving use of new oral anticoagulants for treatment of venous thromboembolism. Blood 2014; 124: $1020-1028$.

6. Agnelli G, Buller HR, Cohen A, Curto M, Gallus AS, Johnson M, et al. Oral apixaban for the treatment of acute venous thromboembolism. N Engl J Med 2013; 369: 799-808.

7. Amin A, Jing Y, Trocio J, Lin J, Lingohr-Smith M, Graham J. Evaluation of medical costs associated with use of new oral anticoagulants compared with standard therapy among venous thromboembolism patients. J Med Econ 2014; 17: 763-770.

8. National Institute for Health and Care Excellence. Final appraisal determination. Apixaban for the treatment and secondary prevention of deep vein thrombosis and/or pulmonary embolism. http://www. nice.org.uk/guidance/gid-tag474/documents/deep-vein-thrombosispulmonary-embolism-treatment-secondary-prevention-apixabanid726-final-appraisal-determination-document 2 (accessed March 13, 2015).

9. Schulman S, Kearon C; Subcommittee on Control of Anticoagulation of the Scientific and Standardization Committee of the International Society on Thrombosis and Haemostasis. Definition of major bleeding in clinical investigations of antihemostatic medicinal products in non-surgical patients. J Thromb Haemost 2005; 3: 692-694.

10. Buller HR, Cohen AT, Davidson B, Decousus H, Gallus AS, Gent $\mathrm{M}$, et al; Van Gogh Investigators. Idraparinux versus standard therapy for venous thromboembolic disease. N Engl J Med 2007; 357: 1094-1104.

11. Writing Committee, Buller H, Deitchman D, Prins M, Segers A; Botticelli Investigators. Efficacy and safety of the oral direct factor Xa inhibitor apixaban for symptomatic deep vein thrombosis: The Botticelli DVT dose-ranging study. J Thromb Haemost 2008; 6: 1313-1318.

12. Rosendaal FR, Cannegieter SC, van der Meer FJ, Briët E. A method to determine the optimal intensity of oral anticoagulant therapy. Thromb Haemost 1993; 69: 236-239.

13. ICH Steering Committee. Ethnic factors in the acceptability of foreign clinical data (ICH-E5(R1)). 1998. http://www.ich.org/fileadmin/ Public_Web_Site/ICH_Products/Guidelines/Efficacy/E5_R1/Step4/ E5_R1_Guideline.pdf (March 13, 2015).

14. Agnelli G, Buller HR, Cohen A, Curto M, Gallus AS, Johnson M, et al. Apixaban for extended treatment of venous thromboembolism. $N$ Engl J Med 2013; 368: 699-708.

\section{Appendix \\ Members of the AMPLIFY-J Study Group \\ Steering Committee}

Hisao Ogawa (Kumamoto University), Mashio Nakamura (Mie University).

\section{Event Adjudication Committee}

Masakatsu Nishikawa (Mie University), Isao Kitajima (University of Toyama), Yoshio Uetsuka (Tokyo Women's Medical University), Takuji Yamagami (Kochi University), Hiroki Minamiguchi (Wakayama Medical University), Rika Yoshimatsu (Kochi University).

\section{Data Safety Monitoring Committee}

Mitsuru Murata (Keio University), Toshiaki Iba (Juntendo University), Yasunori Sato (Chiba University).

\section{Medical Advisor}

Issei Komuro (University of Tokyo).

Pfizer Japan Clinical Core Team

Daisuke Shima, Kosuke Tanabe (Japan Clinical Leader), Sayaka Kobayashi (Clinical Scientist), Kazuhiro Kanmuri (Japan Development Leader), Nobushige Matsuoka (Study Statistician) and Masao Kasaya (Japan Study Lead).

\section{Study Sites Recruiting at Least 1 Patient}

Toho University Sakura Medical Center, Kokura Memorial Hospital, Fukushima Medical University Hospital, St. Luke's International Hospital, Aichi Medical University Hospital, Saiseikai Kumamoto Hospital, Teine Keijinkai Hospital, National Hospital Organization Okayama Medical Center, Hiroshima General Hospital, National Hospital Organization Yokohama Medical Center, Nihon University Itabashi Hospital, Mie University Hospital, Kumamoto University Hospital, National Cerebral and Cardiovascular Center Hospital, Tokyo Medical University Hospital, Yokohama Minami Kyousai Hospital, Kinki University Hospital, Japanese Red Cross Musashino Hospital, Kanazawa Medical University Hospital, National Hospital Organization Tokyo Medical Center.

\section{Supplementary Files}

Supplementary File 1

Table S1. Definition of bleeding events and efficacy endpoints

Please find supplementary file(s); http://dx.doi.org/10.1253/circj.CJ-15-0195 